\title{
Trade-Offs for Wireless Transcutaneous RF Communication in Biotelemetric Applications
}

\author{
Inke Pitz ${ }^{a, b, c}$, Leonard Hall ${ }^{a, b}$, Hedley Hansen ${ }^{d}$, Vijay Varadan ${ }^{e}$, Chris Bertram ${ }^{f}$, Simon \\ Maddocks $^{g}$, Stefan Enderling ${ }^{a, b, h}$, David Saint ${ }^{a, i}$, Said Al-Sarawi ${ }^{a, b}$ and Derek Abbott ${ }^{a, b}$ \\ ${ }^{a}$ Centre of Biomedical Engineering, University of Adelaide, SA 5005, Australia \\ ${ }^{b}$ Department of Electrical and Electronic Engineering, University of Adelaide, SA 5005, \\ Australia \\ ${ }^{c}$ University of Applied Sciences, Department of Biomedical Engineering, Gelsenkirchen, \\ Germany \\ ${ }^{d}$ DSTO, EWD, PO Box 1500, Edinburgh, SA 5111, Australia \\ ${ }^{e}$ Research Center for the Engineering of Electronic and Acoustic Materials, Pennsylvania \\ State University, University Park, PA 16802, USA \\ ${ }^{f}$ Graduate School of Biomedical Engineering, University of New South Wales, NSW 2052, \\ Australia \\ ${ }^{g}$ Department of Animal Science,University of Adelaide, Roseworthy Campus, Roseworthy SA \\ 5371, Australia \\ ${ }^{h}$ Department of Electronics and Electrical Engineering The University of Edinburgh, \\ Edinburgh EH9 3JF, UK \\ ${ }^{i}$ Department of Physiology, University of Adelaide, SA 5005, Australia
}

\begin{abstract}
The application of biotelemetry in case of a RF controllable microvalve is discussed. Biotelemetry implies the contactless measurement of different electrical and nonelectrical parameters measured on human or animal subjects. A biotelemetry system consists of a transmitter and a receiver with a transmission link in-between. Transmitted information can be a biopotential or a nonelectric value like arterial pressure, respiration, body temperature or $\mathrm{pH}$ value. Transducers convert nonelectrical values into electrical signals. Radio frequency (RF) telemetry allows a patient greater mobility. Above all, the application of wireless communication becomes more and more popular in microinvasive surgery. Battery powered implants are most commonly used, but batteries must be changed after a period of time. To avoid this, wireless transcutaneous radio frequency (RF) communication is proposed for the powering and control of medical implants.
\end{abstract}

Keywords: RF communication, SAW, microvalve, microantennas

\section{INTRODUCTION}

Future MEMS systems will require the integration of active as well as passive devices. There are many types of devices produced for different tasks, for example microchannels, micropumps and microvalves. These microstructures (e.g. microfluidic) are divided in two different categories: passive devices (without an actuator) and active devices (with an actuator). ${ }^{1}$

Passive microvalves get their activating energy from the surrounding fluid and flow in only one direction is possible. On the other hand the energy of active valves is externally supplied. The advantage of using active valves is that the control of very small sample volumes is possible, which is very important in biomedical, chemical, drug delivery and publishing applications. They have a small dead volume that allows greater efficiency and makes a fast response time possible. Designs that do not unnecessarily heat the surrounding medium are possible. $^{2}$ The right choice of actuating principle is dependent on the structural dimensions, the technology, the response time, the force or torque as a function of displacement and the maximum power as well. Force can be generated using two main principles:

Biomedical Applications of Micro- and Nanoengineering, Dan V. Nicolau, Abraham P. Lee, Editors 307 Proceedings of SPIE Vol. 4937 (2002) (C) 2002 SPIE · 0277-786X/02/\$15.00 
1) External forces are generated in the space between stationary and moving parts using thermopneumatic and electromechanical effects, electrostatic and magnetic fields or

2) Inner forces that use special materials having intrinsic actuation capabilities including piezoelectric, thermomechanical, shape memory, electro- and magnetostatic effects.

The main principles used for microvalves which have been successfully realized and implemented in industrial applications or prototypes, are electromagnetic, thermodynamic, thermopneumatic and shape memory. ${ }^{3-11}$ In our case the converse piezoelectric effect is used to control the microvalve. Because of surface acoustic wave (SAW) devices, new possibilities in controlling and processing electrical signals have been found. We will present the restrictions we face on antenna size and transmitter power and suggest a minimum suitable size for the internal antenna in across the frequency range of $100 \mathrm{MHz}$ to $100 \mathrm{GHz}$, with regard to a microvalve consisting of a biocompatible silicone based polymer with piezoelectric properties. This microvalve can be used for different applications, e.g., fertility control, flow cytometry, drug delivery and DNA sequencing.

\section{PIEZOELECTRIC EFFECT}

Piezolectricity, discovered in 1880 by the brothers Jacques and Pierre Curie, is defined as a change in electric polarization resulting from a change in applied stress, which is usually referred to as the direct piezoelectric effect. It can be utilized for the localization of devices in inaccessible places. The converse piezoelectric effect is a reciprocal effect where there is a change in strain for a free crystal (or stress for a clamped system) resulting from a change in applied field. Thus, the converse piezoelectric effect is typically used when a material is used as an actuator: Input is a voltage gradient, output a strain. For low fields, there is a linear relationship between strain and electrical field. Reversing the field also reverses the direction of the strain. ${ }^{12}$ A piezoelectric material has the characteristic of being elastic and generating an electric field when a force is applied to it and vice versa. Wave propagation on the surface of the piezoelectric material can thus be launched and detected by metal electrodes on the surface of the material. There are currently many substrates in use for SAW devices. The most commonly used are lithium niobate, quartz and lithium tantalate, which are cut at various angles to the crystalline axes. In our case a piezoelectric polymer is used to activate the microvalve.

\section{SAW DEVICES}

Surface acoustic wave (SAW) devices are recognized for their versatility and efficiency in controlling and processing electrical signals. They are based on propagation of acoustic waves in elastic solids and the coupling of these waves to electric charge signals via electrodes deposited on a piezoelectric material. Basically, we may think of a SAW device as consisting of a solid substrate with an input and output transducer. Because of this interdigital transducers (IDT) provide a practical method for generating and detecting surface acoustic waves. The input transducer converts the incoming signal by the inverse piezoelectric effect into acoustic waves, which propagate along the planar surface of the solid. At the output transducer, the SAWs are reconverted to an electrical signal. A standard Rayleigh SAW device is shown in Figure 1.

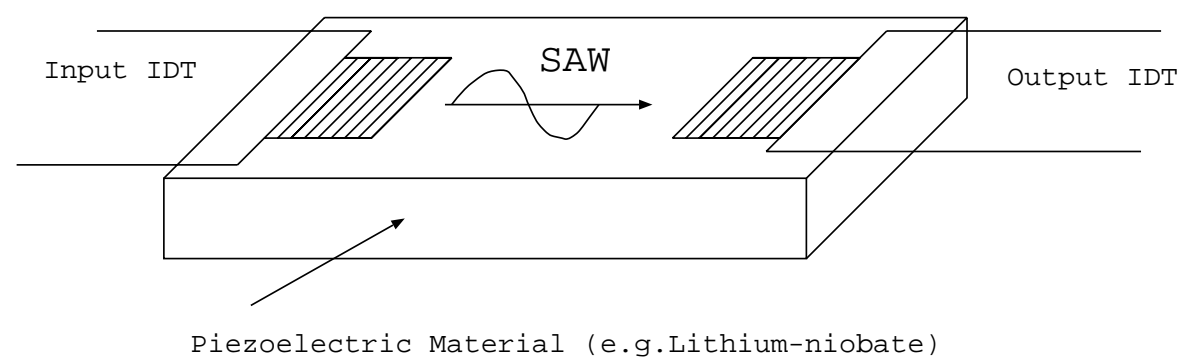

Figure 1. This figure shows a standard Rayleigh surface acoustic wave device with its In- and Output IDTs, and the propagation of the surface acoustic waves. 
A fundamental property of the SAW device is its ability to act as a signal delay line. The relatively slow propagation velocity of the surface acoustic waves of typically $3500 \mathrm{~m} / \mathrm{s}$ allows delays of several microseconds on a small chip. ${ }^{13}$ The phase velocity of the wave combined and the photolithographic resolution of the fabrication process, determine the maximum sampling frequency of the transducers.

As an alternative to the use of a two part SAW device, the receiving section can be replaced by a series of reflectors and the output Identification Device (ID-tag) can be directly connected to a microstrip antenna to receive and transmit RF signals. The transmitted RF signal is received by the IDT antenna and transformed into a propagating surface acoustic wave. The response signals form a sequence of short pulses in accordance with the number and position of the reflectors on the crystal surface. The time delay of every partial response pulse depends on the SAW propagation velocity, which is affected by several physical quantities (temperature, pressure and strain) and the distance between the IDT and the reflectors. ${ }^{14}$ The response signal is reflected to the IDT and transduced back into electromagnetic waves. These waves are radiated by the antenna and received and evaluated by the reader station. So it is possible to get information about the localization of the device as well as the ablility to control its function. The basic principle of a wireless SAW ID-tag is shown in Figure 2 .

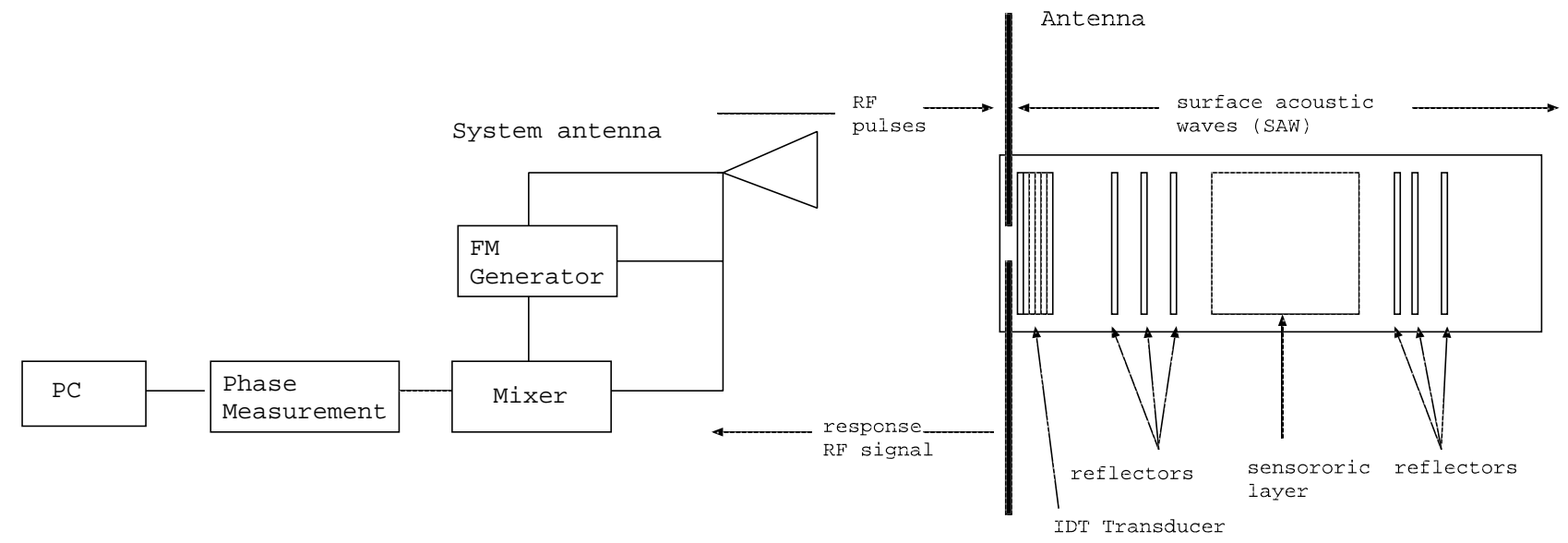

Figure 2. This figure shows the principle of a wireless SAW ID-tag which uses reflectors on its surface instead of an output IDT to reflect the SAWs back to the input IDT which is directly connected to the antenna. This antenna is able to receive as well as to transmit RF pulses. The figure also shows the reader/transmitter station with its system antenna. The reading system has a linear Frequency Modulated (FM) signal generator and the FM signals are transmitted by the system antenna.

Today, mainstream integrated circuits tend to use silicon as the substrate material. However, there is now a new growing field in the area of polymer electronics. This field is moving rapidly now that researchers have successfully fabricated transistors on a polymer substrate. ${ }^{12}$ This new paradigm has the advantage that circuits can be flexible and lightweight. Also the material parameters of a polymer can be more finely tuned than in silicon. $^{12}$ A further advantage is that polymer materials are more bio-friendly for implantation in animals or the human body - this is attractive for a host of applications from artificial valves to pacemakers. Polymer materials can be flexed and textured, whereas silicon is hard and brittle. The active circuitry on a polymer can be elegantly encapsulated and isolated from body fluids and the like.

However, one problem with conventional transistor circuity, even on polymers, is that transistors require a source of power, ie. a battery. The number of useful biomedical applications would greatly increase, if a battery was not needed. There are obvious disadvantages with implanting batteries into the body - as well as the problem of replacement, the extra mass means that it is only limited to special applications such as in pacemakers.

A possible solution is to use conventional radio frequency identification device (RFID) technology where low power circuits can obtain their power from an electromagnetic field. However, there are problems here: (a) the resulting circuit size would be quite large for, say, application in male fertility control, (b) full integrated circuit 
technology on polymer is not mature enough yet and (c) there is no direct actuation mechanism in this type of technology.

Our proposed solution is to exploit an exciting new development in making passive circuits on polymers (where "passive," here, means no battery power). This solution exploits the principle of a Surface Acoustic Wave (SAW) device on a polymer substrate. The SAW device is a set of interdigitated conducting fingers on the polymer substrate. If an appropriate radio frequency $(\mathrm{RF})$ signal is sent to the device, the fingers act as microantennas that pick up the signal, and this energy is then converted into acoustic waves that travel across the surface of the polymer substrate. Being a flexible polymer, the acoustic waves cause stresses that can either contract or stretch the material. In other words, we effectively have an RF to strain transducer. In an appropriately designed form, this principle has been successfully used to continuously monitor the stresses on helicopter rotor blades. ${ }^{15}$ There is no battery required in the strain sensor as all the energy comes from the RF field - moreover, the system is completely wireless as the strain measurements are obtained from the interrogating RF signal. Other wireless strain sensors using this principle have been successfully reported. ${ }^{16-22}$

\section{STRUCTURE OF THE MICROVALVE AND WORKING PRINCIPLE}

When the surface acoustic waves cause stresses in the polymer substrate, the resulting deflection can be in the order of microns, ${ }^{18}$ suggesting an ideal novel application would be a microvalve. Essentially, a small hole in the polymer substrate can be stretched open wider or contracted by application of the RF signal on a SAW-on-polymer device. By sandwiching a number of these microvalves together, and by operating them in different phases, a peristaltic micropump could be created - this is very useful in cases where a fluid needs to be "pushed" through the orifice. Alternatively, the SAW could be used to actuate a V-groove valve arrangement (also called a 'check valve'). A further possibility is for passive venous valves (such as in artificial heart valves) to use the polymer device for monitoring the open/shut status of the valve, rather than for actuation. A host of lucrative applications from valves for electronic fertility control to micropumps for nanolitre drug delivery become foreseeable. The basic structure of the V-groove microvalve is shown in Figure 3.

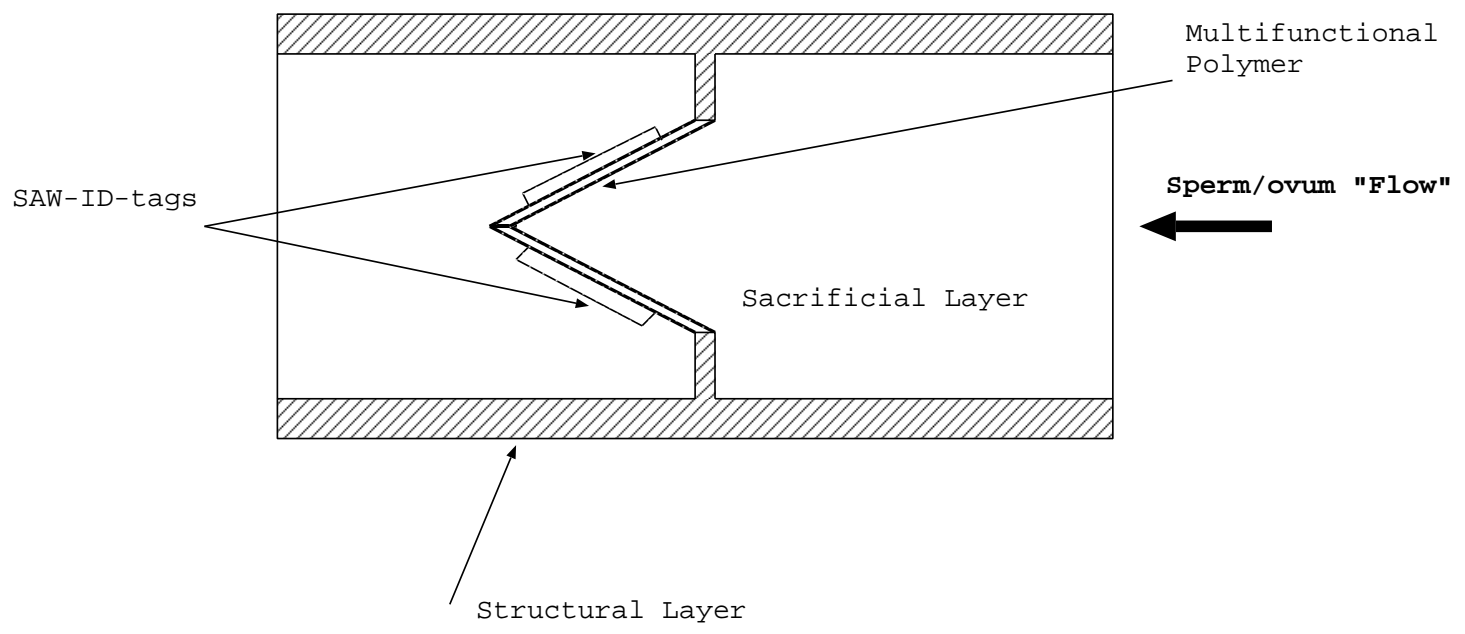

Figure 3. This figure shows the basic structure of a V-groove microvalve enhanced by SAW tags. It can be a passive valve or an active one. The passive microvalve can be opened or closed by the pressure of the surrounding fluid depending on the direction of the fluid stream. In this example the valve will be opened by the pressure. In the case of a passive microvalve the SAW ID-tag electrodes have only the function of monitoring the status of the valve. That means that they are sending an RF signal to a reader station to give information about whether the valve is open or closed and where it is localized. But the microvalve can also be used as an active one. In this case the SAW ID-tag electrodes work as actuators stretching open the polymer flaps.

Three different polymer layers are utilized to create the valve, (a) UV curable structural polymer to form the body of the valve, (b) a sacrificial polymer that can be etched away to leave cavities in the resulting 3D structure 
and (c) a multifunctional polymer that has piezoelectrical properties that can act as either a SAW sensor or actuator. The structural polymer, UV 001, is from HVS Technologies. It is a UV curable polymer with urethene acrylate, epoxy acrylate and acryloxysilane as the main ingredients. Its low viscosity allows easy processing through automatic equipment or manual methods without the addition of solvents or heat to reduce the viscosity. It also complies with all VOC regulations. It has excellent flexibility and resistance to fungus, solvents, water and chemicals. The sacrificial polymer is essentially an acrylic resin containing $50 \%$ silica. This composition can be dissolved with $2 \mathrm{Mol} / \mathrm{L}$ caustic soda at $80^{\circ} \mathrm{C}$. The multifunctional layer consists of nanoceramic particles attached by chemical bonding as side groups on a polymer backbone. The concept is a backbone with functional groups that will serve as anchor points for nanoparticle metal oxides. Nanoparticles such as PZT, PLZT, etc. must have active surfaces or functional groups that can bond with the polymer chain. The nanoparticles provide the piezoelectric function in the polymer and the backbone provides mechanical strength and structural integrity, electrical conductivity, etc. The backbone can be the same type of polymer as used for the structural layers. A new class of functional polymers have been synthesized at Penn State, which can serve as the host for piezoelectric actuation.

\section{ANTENNA CALCULATIONS}

To receive and transmit the RF signal a specific antenna is required. Because the microvalve should be implanted in the body there are many things to be observed. We assumed the antenna should deliver a max. power of 20 $\mu \mathrm{W}$ (as will be explained later) and must not be larger than $10 \mathrm{~cm}$. The length $l$ has been calculated by the following equations, assuming a simple dipole antenna:

$$
\begin{gathered}
\lambda=\frac{c}{f} \\
l=\frac{\lambda}{x} \Rightarrow l=\frac{c}{x f}
\end{gathered}
$$

The relationship between the used frequency and the resulting antenna size is shown in Figure 4(a) and (b).

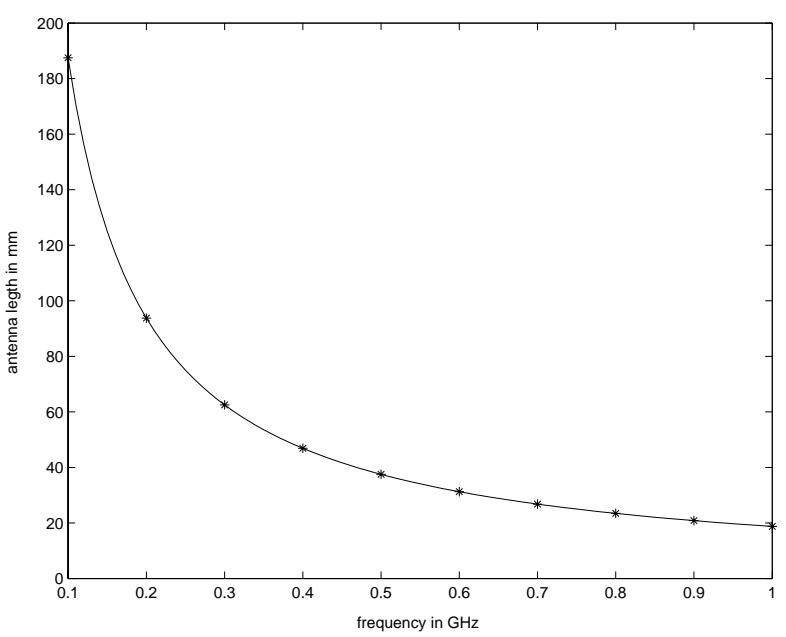

(a) Relation between the frequencies $0.1-1 \mathrm{GHz}$ and the antenna size

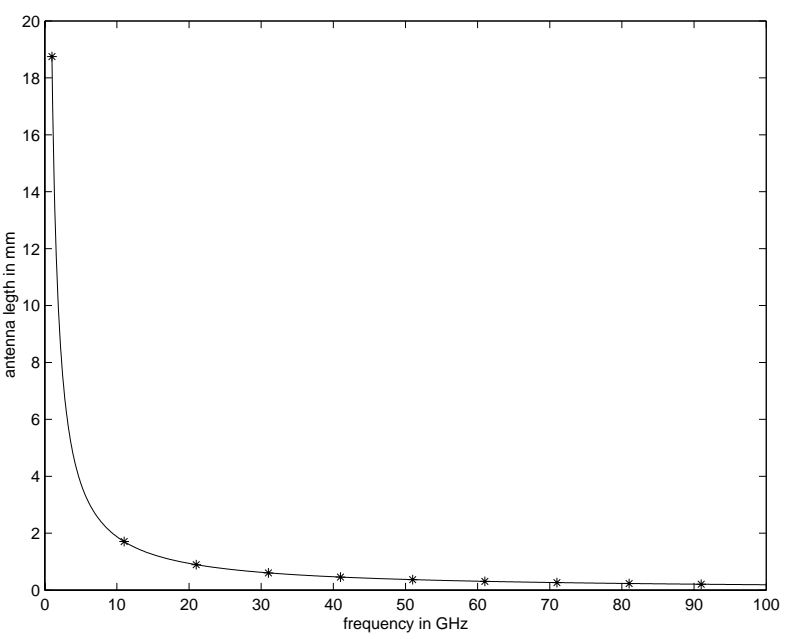

(b) Relation between the frequencies $1-100 \mathrm{GHz}$ and the antenna size

Figure 4: Antenna Size for $x=16$

For the calculation we assumed $x=16$, used the frequencies $f$ in the $1 \mathrm{MHz}-100 \mathrm{GHz}$ range, the velocity of light in free space $c=3 \times 10^{8} \mathrm{~m} / \mathrm{s}$ and the wavelength $\lambda$. 
But before we can choose a suitable size for the antenna, there is another problem: The attenuation due to human body tissue. But neither the electric field (E) nor the magnetic field (H) penetrate far into a "good" conductor. The point where the fields are reduced by a factor of $1 / e \sim 1 / 2.71$ is called the skin depth.

Actuation forces (including external load) required for such a microvalve can be typically of the order of 100 $\mu \mathrm{N}^{23}$ and moving through, say, a $10 \mu \mathrm{m}$ displacement, corresponds to $1 \mathrm{~nJ}$ of energy.

For our calculations of the maximum power density $S$ that is allowed to be used we assumed the worst case of $100 \mathrm{~nJ}$ of energy. If the SAW device aperture is oscillating in $10 \mathrm{~Hz}-100 \mathrm{~Hz}$ we arbitrary pick $50 \mathrm{~Hz}$. So the maximum delivered power of the antenna must be $20 \mu \mathrm{W}$. We also assumed an effective area of the antenna of about $1 \mathrm{~cm}^{2}$. We calculated the decrease of the inserted power density by equation (3) and the relationship between this power density and its penetration depth at four different frequencies is shown in Figure 5 .

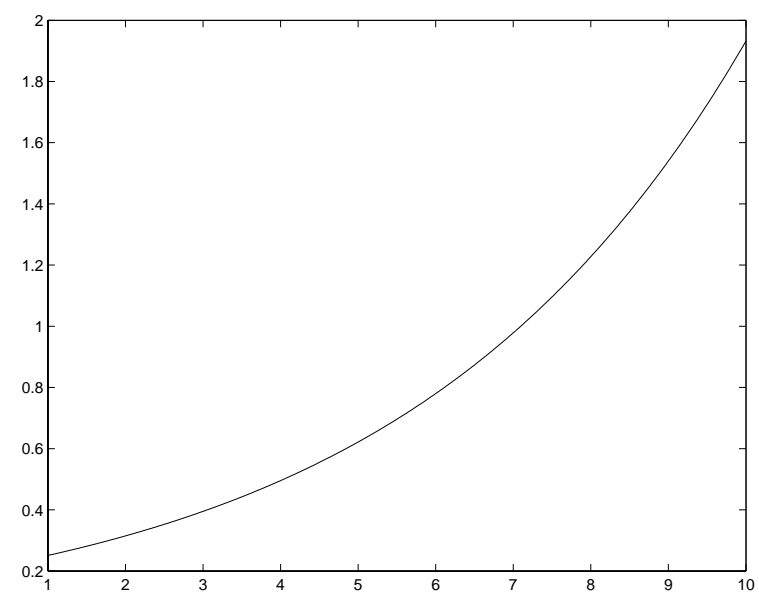

(a) transmitted power density at $600 \mathrm{MHz}$

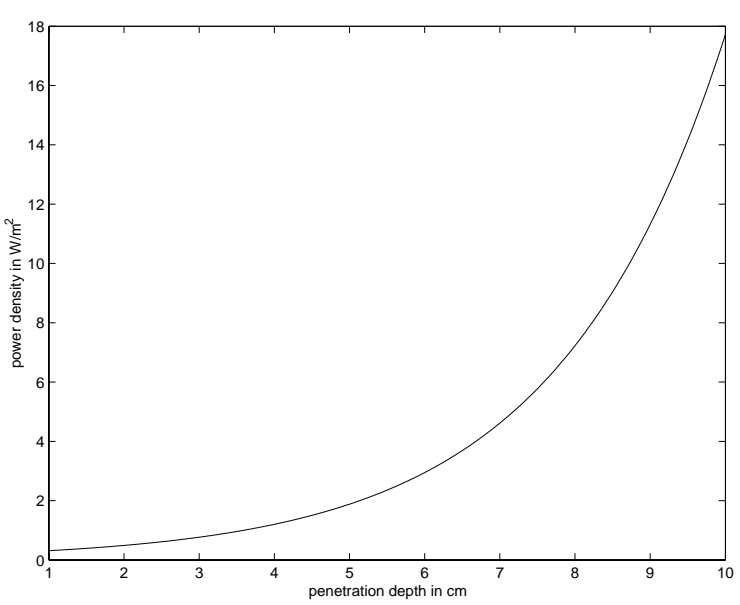

(c) transmitted power density at $2.45 \mathrm{GHz}$

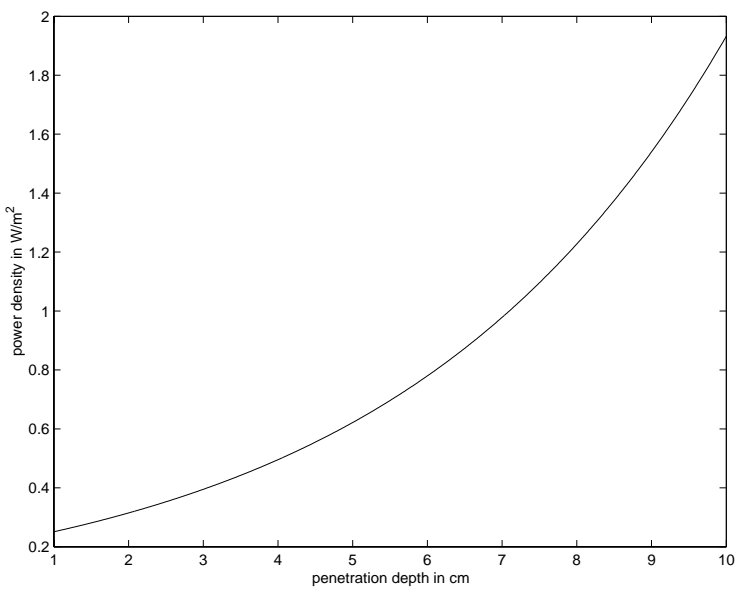

(b) transmitted power density at $800 \mathrm{MHz}$

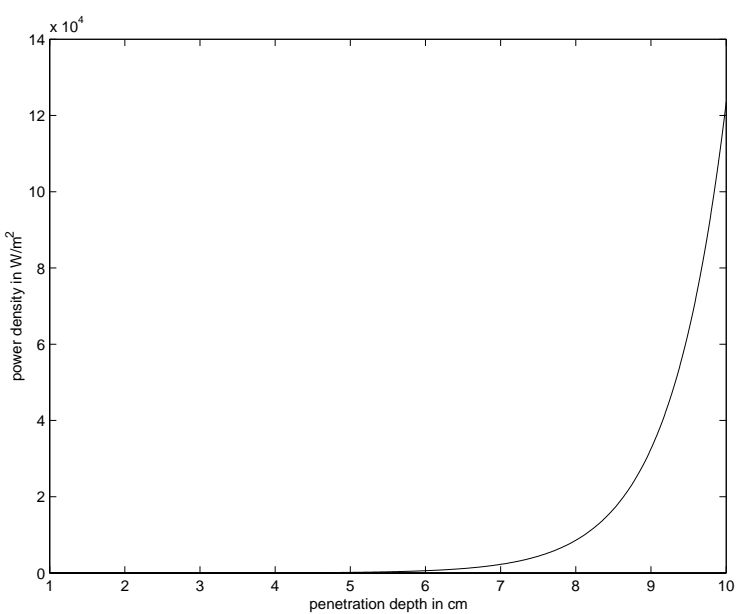

(d) transmitted power density at $5.8 \mathrm{GHz}$

Figure 5. These graphs show the different power densities which will reach the surface of the human body at four different frequencies and for different thicknesses of the human tissue to achieve given penetration depth. In this case we used the absorption coefficient $\alpha=1 / \delta$ where $\delta$ is the penetration depth for muscle at the frequencies $600 \mathrm{MHz} \delta=67.57 \mathrm{~mm}$, $800 \mathrm{MHz} \delta=44.09 \mathrm{~mm}, 2.45 \mathrm{GHz} \delta=22.30 \mathrm{~mm}$ and $5.8 \mathrm{GHz} \delta=7.50 \mathrm{~mm}^{24}$ 
Where $S$ is the power density that should reach antenna on the valve, here $S=0.2 \mathrm{~W} / \mathrm{m}^{2}, S_{0}$ is the power density which has to be transmitted, $x$ is the thickness of the human tissue and $\delta$ is the penetration depth as a function of frequency.

$$
S=S_{0} * e^{-x / \delta}
$$

We assumed that the microvalve needs a power of about $20 \mu \mathrm{W}$ to change its state and an effective area of the antenna of about $1 \mathrm{~cm}^{2}$ which would be approximately realistic for a valve implanted in some kind of vessel in the human body. So the power density we need for this antenna has to be $0.2 \mathrm{~W} / \mathrm{m}^{2}$. At a frequencies between $400-2000 \mathrm{MHz}$ a power density of $f / 40 \mathrm{~W} / \mathrm{m}^{2}$ is allowed ${ }^{25}$ to reach the body surface. Here, $f$ is the RF in $\mathrm{MHz}$.

\begin{tabular}{|l|l|}
\hline Frequency range & $S_{0}\left(\mathrm{~W} / \mathrm{m}^{2}\right)$ \\
\hline $600 \mathrm{MHz}$ & 15 \\
\hline $800 \mathrm{MHz}$ & 20 \\
\hline $2.45 \mathrm{GHz}$ & 61.25 \\
\hline $5.8 \mathrm{GHz}$ & 145 \\
\hline
\end{tabular}

Table 1. Reference levels for occupational exposure to time-varying electric and magnetic fields from the International Commission on Non-Ionizing Radiation Protection (ICNIRP). ${ }^{25}$ So is the power density incident on the human body.

Figures 5(a)-(d) and Table 1 show that the power density at a frequency of $5.8 \mathrm{GHz}$ is too high to be used in our case. The frequencies $600 \mathrm{MHz}, 800 \mathrm{MHz}$ and $2.45 \mathrm{GHz}$ would be allowed to be used for an antenna with an effective area of $1 \mathrm{~cm}^{2}$ and a suitable power density of $0.2 \mathrm{~W} / \mathrm{m}^{2}$ incident on the device.

\section{APPLICATIONS}

This section describes some of the main fields in which the RF controlled microvalve may be applied, i.e., 1) fertility control, 2) drug delivery, 3) flow cytometry and 4) DNA sequencing.

\subsection{Fertility Control}

There are a variety of methods for conception control, i.e., using the "pill" or performing a vasectomy. But the choice of a suitable method is very difficult and the potential risks and benefits must be carefully analyzed. ${ }^{26}$ Because, for example, there are many side effects to using the "pill" and a vasectomy reversal has a high failure rate containing a painful surgical procedure with an inconvenient recovery time for the patient. ${ }^{27}$ To avoid this the microvalve described above could be used. It could be placed in the vas deferens of a male to make restored fertility possible and relatively easy. Within the first two weeks after the insertion the valve can be episodically switched on and off, reducing the backpressure during the early stages when the valve adheres to the walls of the vas deferens and to reduce any possible build up of proteins on the face of the valve. The insertion can be performed by a hypodermic needle. ${ }^{28}$

We are unable to insert the microvalve in the female ovarian duct because the ovarian duct has an inner surface which is coated with microvilli that are like microscopic fingers which help the ova to move along the duct in the correct direction. The ovarian duct is also very sensitive to foreign objects which tends to cause adhesion within the tube. ${ }^{28}$

\subsection{Drug Delivery}

In many different cases is it very useful, when drugs can be delivered at the right time and as close to the treatment area as possible, ${ }^{29}$ as for example, in the case of diabetes mellitus. To make this possible, drug delivery micropumps that can be implanted in the body are used to deliver small quantities of drug from a reservoir. These micropumps would avoid the strain of weekly or daily injections. In this case, a RF controlled microvalve could be integrated in an implanted drug delivery system and make it possible to set the correct drug dosage to be applied at the right time. ${ }^{30}$ Existing approaches either use an osmotic valve, which unfortunately provides a continuous feed, or have the disadvantage of battery power. 


\subsection{Flow Cytometry}

The basis of flow cytometry is that specific optical characteristics (e.g. fluorescence, light scatter) can provide a measure of the specific physical or chemical properties of biological particles (e.g. size and DNA content). The principle of flow cytometry has been integrated into a micromachined silicia flow chamber. ${ }^{31}$ In this instrument a collection of small particles is pumped through a specially designed transparent tube. A measurement is made when the particles pass the "sensoring region". This region is delimited by the illumination and collection regions which are provided by light source and optical detector assemblies.

The RF controlled microvalve could be integrated in a flow cytometry system to control the stream of particles, which would keep the biological cells of interest in a separate particle chamber for later investigations. It could also prevent a reverse flow of the cells out of this chamber. ${ }^{30}$

\subsection{DNA Sequencing}

Present DNA sequencing methods employ electrophoresis and it has taken nearly 20 years to map the whole human genome. To speed up the mapping process, one idea is to pull a strand of DNA through a tiny hole in a polymer material immersed in an ion solution. ${ }^{32}$ The variations of the ion current are detected, which are related to the $\mathrm{A}, \mathrm{G}, \mathrm{C}$ or $\mathrm{T}$ regions of the DNA molecule. One key problem is that of poor signal-to-noise ratio (SNR) in the measured current. The proposed SAW-on-polymer devices provides a means to adaptively modulate the diameter of the hole to optimize the SNR, via use of lock-in amplifier techniques.

\section{FERTILITY CONTROL MODELLING STUDY}

The microvalve which can be employed for fertility control or to replace a vasectomy is shown in Figure 6 . The main element of the valve is the multifunctional polymer actuator, which serves at the same time as a closure element of the microvalve. The wireless communication principle via ID-tag is used as previously described. The ID-tag is deposited on the surface of the polymer actuator. The corresponding reader/transmitter station is outside the human body. The valve is closed in the initial state if no RF signal is sent to the ID-tag. In this state, infertility is set. If a sperm or ovum should pass through the microvalve, RF pulses are transmitted by the base station, which is located outside the body, to the valve. Because of the deformation of the actuator material, the microvalve opens and closes with a certain frequency, which depends on material damping and the metal electrode configuration on the SAW device. Static opening is difficult, so the intention is to develop a device in which the orifice opens cyclically at a certain frequency. The lower the frequency, the more severe the demands on the RF power to the device. On the other hand, as will now be examined, there are fluid-dynamic restrictions on how high the frequency can be.

So, let us now consider the possible simplifications for modelling the fluid flow through the oscillating orifice. The orifice must not vibrate so fast that one sperm cannot get through in one cycle (this restriction can be defined by a Strouhal number). To get an idea of the sizes we are talking about the head of a typical human sperm has a length of 5-6 $\mu \mathrm{m}$, the diameter (width) is $2.5-3.5 \mathrm{mum}$ and the width/length ratio is $1 / 2-3 / 5{ }^{33}$ There is also the possibility of damage to the sperms. The achievable rate will also drop dramatically when the opening is loaded by surrounding fluid. The situation has some similarity to that of a vibrating diaphragm for a pressure transducer; the natural frequency goes down by at least two orders of magnitude when a liquid-filled catheter is connected. Alternatively, if the valve were so powerful that it vibrated without being slowed down by the fluid, it would at some frequency cavitate the fluid, with disastrous effects on valve longevity and (again) degree of sperm damage. This effect is well known from ultrasonic frequencies at therapeutic power levels.

For the purposes of initial analysis of the problem, one can ignore geometric details, and examine for instance an oscillating aperture. However even that simplification poses a spectrum of possibilities between on the one hand a hole time-varying radius in a wall of negligible thickness and on the other a long pipe of time-varying radius through a very thick wall. These present completely different difficulties for fluid flow.

Therefore a still further level of simplification is addressed. The approach is to think of this interrupted flow (geometry unspecified, but zero-thickness shutter is probably closest in spirit) as having to accelerate from zero every time the shutter opens, under the action of the prevailing pressure gradient as set by whatever pressures are exerted upstream and downstream. On this basis the flow is not unlike the situation of a purely oscillatory 
flow in a pipe, under the influence of a sinusoidal pressure gradient. The assumed flow is then laminar and Newtonian, and takes place far from the ends of a straight pipe. This is a standard problem, for which the solution was provided by Womersley (1957). ${ }^{34,35}$ Inserting the fluid properties (density and viscosity of water) and tube diameter of the vas deferens $(0.4 \mathrm{~mm}$ internally) leads to the result shown (Figure 6 ). The result may favorably err on the conservative side, in that there is no allowance for the elastic storage of energy in the vessel wall upstream of the shutter when it closes.

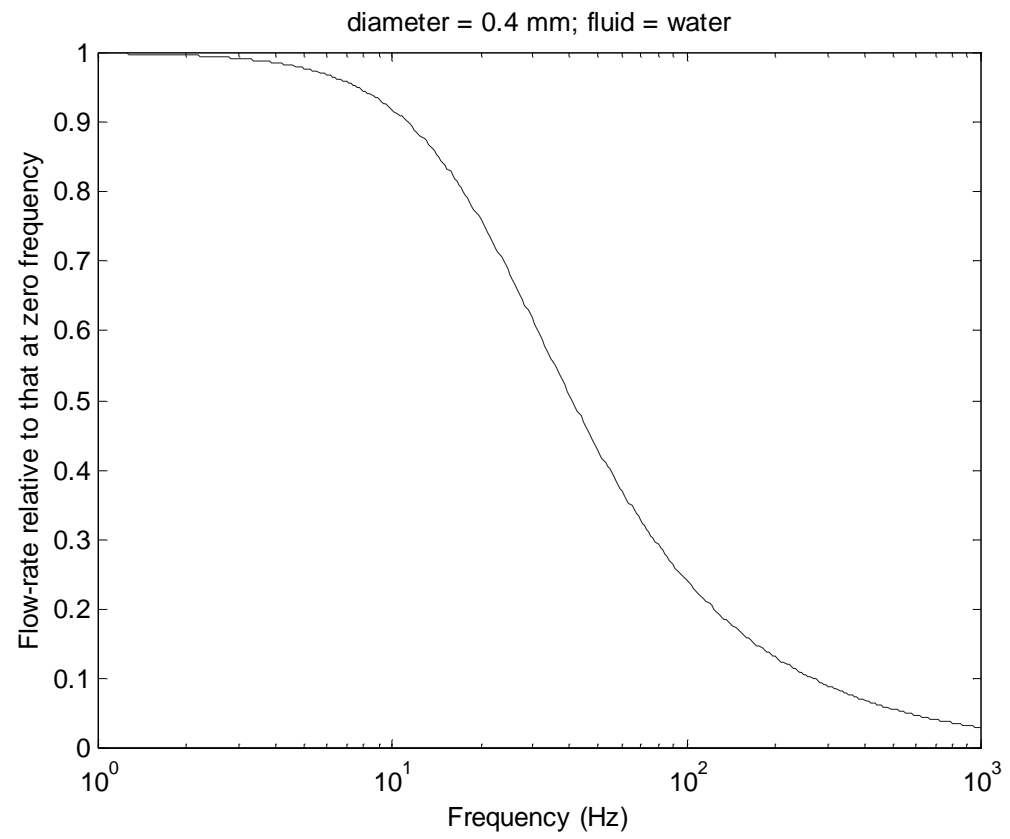

Figure 6. Normalised flow-rate vs. frequency of valve switching. As the orifice frequency increases from 1 to $1000 \mathrm{~Hz}$, the impedance to one-way aqueous flow rises, such that normalised flow-rate is reduced to $10 \%$ or less above $250 \mathrm{~Hz}$.

The decline of oscillatory flow-rate with frequency indicates that despite the small tube diameter, in the frequency range of interest the situation is far from being purely Stokes flow; momentum plays a highly significant role in determining the flow that occurs in response to the applied pressure gradient. In the present context, what is here determined is the extent of forward flow for a given frequency of opening orifice, relative to the flow that would occur through the tube if the microvalve presented no obstruction at all by periodic closure. It is also assumed that no partial obstruction of the tube occurs when the microvalve is open.

The fluid dynamic simulation has a typical low pass characteristic with a cut off frequency $f_{c}$ of $30 \mathrm{~Hz}$. The frequency range of interest here is between $10 \mathrm{~Hz}$ and $100 \mathrm{~Hz}$. In that particular range, the flow-rate relative to that at $0 \mathrm{~Hz}$ is $90 \%$ for $10 \mathrm{~Hz}$ and $25 \%$ for $100 \mathrm{~Hz}$. From the physiological point of view, the flow rate at $100 \mathrm{~Hz}$ is sufficient for the sperm transport through the microvalve, whereas it is desirable to have a higher flow-rate. The flow simulation shows that a material is needed which is able to oscillate in that frequency range. Oscillations at such low frequencies are achieved by a special arrangement and distances of electrodes on the surface of the SAW device and by the fact that the nano-particle embedded polymer is a highly damped material. Frequencies down to $100 \mathrm{~Hz}$ have been achieved at Penn State and work is ongoing for further reduction.

\section{OPEN QUESTIONS AND FUTURE WORK}

We considered a number of application areas for a RF controllable microvalve in this paper. In new fields of research, there are many questions to be answered before any invention can be safely applied. Above all, in the field of fertility control it is important to make sure that the microvalve works correctly and efficiently to prevent problems which could endanger the patient. 
Considering the expansion of the actuator material, analysis should also be done if a particular shape of the microvalve has an influence on the displacement of the actuator material. It is not yet known, whether there exist differences in displacement of different shapes of the valve. The aim is to find the "optimum" shape which satisfies the requirements of displacement for application in the area of fertility control. Furthermore, it should be established which shape is the easiest to implant in the body in order to prevent complications in surgery and additional pain for the patient. Initially, these tests, together with tests for the functionality of the valve can be undertaken in animals such as sheep or pigs.

Apart from that point of view, there is also room for electromechanical modeling of the actuator material of the valve. Especially the gain of the antennas versus the suitable frequency ranges has to be carefully analyzed. In this paper we discussed a rough survey of the frequencies and the power densities which are allowed to be used. The next step in research is to analyze the efficiency of the antenna, because the lower the RF frequency, the lower the gain of the antenna, which makes it difficult to deliver enough power to cause a certain amount of strain in the material. In this study we only considered simple dipole antenna structure. Improved trade-offs between efficiency and antenna size may be achieved by use of other geometries such as fractal antennas. ${ }^{36}$

For a given frequency, sub-optimal small antennas may be possible at the expense of increased external RF power - the limit to this trade-off will depend on the safety requirement for transcutaneous RF transmission for each given application. Given that the human vas deferens is of the order of $400 \mathrm{~m}$ in diameter, male fertility control is a rather ambitious goal, as the whole integrated valve would have to fit into that diameter. Therefore our near-term goal will be to firstly target drug-delivery applications, where the size restriction can be greatly eased. Size requirement will impact on the lowest usable RF frequency, and in this program we will push valve size and antenna size down as far as we can go to find the limits. The ultimate goal of a $400 \mathrm{~m}$ size for male fertility control is not untenable, however. To achieve this would require high a dielectric layer on a chiral absorber, using a fractal microantenna approach. Note also that the space saving by using the Sierpsinki fractal approach of Puente ${ }^{36}$ has recently been surpassed by using a Hilbert curve fractal. ${ }^{37}$ Although bringing the concept of the Hilbert fractal antenna together with the chiral absorber is beyond the scope of this proposal, we will examine its feasibility for a future phase.

Another question is whether the SAW device can exert the forces required to control flow against ultimately physiological pressure gradients. In the short term, the proposed device will simply be limited to a narrow range of applications, if higher pressure gradients prove problematic. In the longer term, new polymer substrate materials may emerge (e.g. nanotube embedded polymer) that exhibit higher actuation strains.

The aim of material simulations is to find a material which needs a very low energy level to cause a relative high deformation. The frequency behavior of the material could be calculated by a simulation of the valve. Together with a simulation of the different shapes of microvalves, we can optimize microvalve set-up for fertility control. As a result of these simulations, a first model could be constructed following the required design specifications.

In modelling the SAW device, different acoustic modes need to be analyzed - for example, one would expect horizontally polarized shear modes to be of more importance than Rayleigh modes.

\section{CONCLUSION}

In this paper a RF controllable microvalve for medical applications was discussed. By using wireless communication and surface acoustic wave devices, a valve can be devised for implantation into a section of the vas deferens that occurs just after the epididymis. The implantation can be done by use of a hypodermic needle.

For implantation the valve material has to be compatible with the human body. The microvalve which is described in this paper consists of silicone based polymers, which are expected to be biocompatible. In principle, the biocompability issue can in future be further addressed by use of dacron or biopolymer silicone coatings on the valve.

The working principle of the microvalve is based on the previously described SAW concept in which a reader/transmitter station (outside the body) sends RF pulses to an ID-tag (inside the body), which is printed on the actuator of the valve. These RF pulses are received by the ID-tag of the valve and converted into Surface Acoustic Waves (SAWs). The SAWs propagate along the surface of the piezoelectric actuator and cause the required alternating deflection which is used to open and close the valve at a certain frequency. 
To receive the RF signal the ID-tag is directly connected to an antenna. In this paper the size of the antenna, suitable frequencies and the required power density were discussed. First we calculated the antenna size for different frequencies to get a rough idea of the required frequency range, assuming an effective antenna area of $1 \mathrm{~cm}^{2}$. We calculated the required transmitted power density for four different frequencies, $600 \mathrm{MHz}, 800 \mathrm{MHz}$, $2.45 \mathrm{GHz}$ and $5.8 \mathrm{GHz}$. After comparing these results with the reference levels of the ICNIRP we were able to eliminate frequencies above $5.8 \mathrm{GHz}$.

The fluid dynamic behavior of the microvalve was discussed in the case study for the fertility control application, where the flow-rate through the oscillating valve aperture has a low pass characteristic. The frequency range of interest is between $10 \mathrm{~Hz}$ and $100 \mathrm{~Hz}$. These frequencies are achieved by a special arrangement of surface electrodes on the actuator material of the valve and arranging suitable acoustic damping in the polymer. So far acoustic frequencies as low as $100 \mathrm{~Hz}$ have been achieved at Penn State. The flow-rate of sperm through the microvalve still obeys physiological requirements for the fertilization of the human ovum in the frequency range of $10-100 \mathrm{~Hz}$. The flow-rate in this range relative to the flow-rate of $100 \%$ at $0 \mathrm{~Hz}$ is $75 \%$ at $100 \mathrm{~Hz}$ and only $10 \%$ at $10 \mathrm{~Hz}$. The next step in research is to simulate the material behavior for SAW devices at such low frequencies.

\section{ACKNOWLEDGMENTS}

The authors would like to thank all members of the Department of Electrical and Electronic Engineering and the Centre of Biomedical Engineering (The University of Adelaide) for their helpful support.

\section{REFERENCES}

1. A.D. Johnson, E.J. Shahoian "Progress in thin film shape memory microactuators," http://www.smamems.com/recent.htm

2. W. van der Wijngaart "Microfluidics-hydraulicsystems on chip," Microsystem Technology, S3, KTH.

3. B.W. Silicon "Microactuators: Activation Mechanisms and Scaling Problems," 6'th Int. Conf. Solid-State Sensors and Actuators, pp. 46-59, 1991.

4. van de Pol FCM, van Lintel HTG, Elwenspoek M., Fluitman JHJ. "A Thermopneumatic Micropump Based on Micro-engineering Techniques," Sensors and Actors, Vol. 21, pp. 198-202, 1990.

5. S. Boehm, W. Olthuis, P. Bergveld 'An Electrochemically Actuated Micropump for Use in a 'Push-Pull' Microdialysis Based In-Vivo Monitoring System," 10th International Conf. on Solid-state Sensors and Actuators, Sendai, Japan, pp. 880-881, 1999.

6. W.S.N. Trimmer, K.J. Gabriel, R. Mahadevan "Silicon Electrostatic Motors," 4th Int. Conf. S.-S. Sensors and Actuators, pp. 857-860, 1987.

7. B. Wagner, M. Kreutzer, W.Benecke, "Linear and Rotational Magnetic Micromotors Fabricated Using Silicon Technology," Proc. IEEE Micro Electro Mechanical Systems, Travemuende Germany, pp. 183-189, 1992.

8. W.P. Robbins, D.L. Polla, T. Tamagawa, D.E. Glumac, "Design of Linear-Motion Micrpactuatoes Using Piezoelectric Thin Films;" J. Micromech. Microeng. 1(4): pp. 247-252, 1991.

9. L.Que, J.S. Park, Y.B. Gianchandani, "Bent-Beam Electrothermal Actuators for High Force Applications," IEEE MEMS, Orlando USA, pp. 31-36, 1999.

10. D. Reynaerts, J. Peirs, H. van Brussel, "An Implantable Drug-Delivery System Based on Shape Memory Alloy Micro-Actuation," Sensors and Acttors, A 61: pp. 455-462, 1997.

11. E. Quandt, K. Seemann, "Fabrication and Simulation of Magnetostrictive Thinfilm Actuators," Sensors and Acttors, A 50: pp. 105-109, 1995.

12. D. de Leeuw, "Plastic electronics," Physics Today, March, pp. 31-34, 1999.

13. C.W. Ruppel, Munich/Germany and T. Fjildly, Kjeller/Norway "Advances in SAW Tchnology, Systems and Applications," vol. 1, 2, pp. 1-3, 2000.

14. F. Schmidt, O Sczesney, C. Ruppel, and V. Magori, "Wireless Interrogator System for SAW-IdentificationMarks, and SAW-Sensor Components," IEEE International Frequency Control Symposium, No. 96CH35935, pp. 208-215, 1996. 
15. H. Subramanian, "Design and fabrication of wireless remotely readable MEMS based microaccelerometers," Smart Materials and Structures, Vol. 5, pp. 730-738, 1997.

16. V.V. Varadan, "Wireless passive IDT strain microsensor," Smart Materials and Structures, Vol. 6, pp. 745751, 1997.

17. V.K. Varadan, "Integration of interdigital transducers, MEMS and Antennas for Smart Structures," Proc. SPIE, Vol. 2722, pp. 95-106, 1996.

18. V.V. Varadan, "IDT sensors for detection of ice on rotorcraft," Proc. SPIE Smart Electronics and MEMS, Vol. 3328, San Diego, pp. 49-58, March 1998.

19. F. Schmidt, O. Sczesney, C. ruppel and V. Magori, "Wireless interrogator system for SAW-identificationmarks and SAW-sensor components," Proc. 1996 IEEE International Frequency Control Symposium (Cat.No.96CH35935), IEEE, New York, NY, USA; pp. 208-215, 1996.

20. V.K. Varadan, "Design and fabrication of wireless remotely readable MEMS accelerometers," in Smart Mater. Struct., vol. 6, pp. 730-738, 1997.

21. V.K. Varadan, "Smart tongue and nose," Proc. SPIE, Vol. 3673, pp. 67-92, March 1999.

22. K. Ikuta and K. Hirowatari, Proc. IEEE International Workshop on Micro Electro Mechanical Systems, pp. 42-47, 1993.

23. M. Kohl, J. Gottert and J. Mohr, "Verification of the micromechanical characteristics of electrostatic linear actuators," Sensors and Actuators, Vol. 53, 1996, pp. 416-422.

24. W.G. Scanlon and N.E. Evans "Numerical Analysis of Bodyworn UHF Antenna Systems," Electronics Es Communication Engineering Journal, p. 56, April 2001.

25. ICNIRP Guidelines, "Guidelines for limitted exposure to time-varying electric, magnetic, and electromagnetic fields (up to $300 \mathrm{GHz}$ )," International Commission on Non-Ionizing Protection, Health Phys. 75(4):442; pp. 509-511, 1998.

26. F. Martini, Anatomy and Physiology, ch. 21,28, Prentice Hall, Englewood Cliffs, New Jersey, 2nd ed., pp. $669,950,1992$.

27. H. Fisch "The patient' s guide to vasectomy reversal," http://cpmcnet.columbia.edu/dept/urology/vasrev.html.

28. D. Moffett, Human Physiology: Foundations and Frontiers, ch. 24, Mosby, 2 ed., p. 688, 1993.

29. P. Dario, M.C. Carrozza, A. Benevenuto, and A. Menciassi, "Microsystems in Biomedical Applications," Micromech. Microeng. 10, pp. 235-244, 2000.

30. S. Enderling, Derek Abbott, Vijay K. Varadan, Karl F. Boehringer, Editors, "Directions for RF controlled intelligent microvalve," Smart Electronics and MEMS 2 Proceedings of SPIE Vol.4236, pp. 204-212, 2001.

31. D. Sobek, S. D. Senturia, and M.L. Gray, "Microfabricated Fused Silica Flow Chambers for Flow Cytometry,", Solid-State Sensors and Actuators Workshop, pp. 260-263, June 1994.

32. J. Kasianowicz, E. Brandin, D. Branton, and D. Deamer, "Characterization of Individual Polynucleotide Molecules Using A Membrane Channel," Proc. Natl. Acad. Sci., 93(24), USA , pp. 13770-13773, 1996.

33. T.F. Kruger, A.A. Acosta, K.F. Simmons, R.J. Swanson, J.F. Matta and S. Oehninger "Predictive value of abnormal sperm morphology in in vitro fertilization," Fertil. Steril.49, pp. 112-117, 1988.

34. J.R. Womersley, "An elastic tube theory of pulse transmission and oscillatory flow in mammalian arteries," Tech. Rep. TR 56-614, Aeronautical Research Laboratory, Wright-Patterson Air Force Base, Ohio, USA, 1957.

35. J.R. Womersley, "Oscillatory flow in Ateries: the constrained elastic tube as a model of arterial flow and pulse transmission," Physics in Medicine and Biology 2(2), pp. 178-187, 1957.

36. C. Puente, J. Anguera, C. Borja and J. Soler, "Fractal-shaped antennas and their application to GSM 900/1800," J. Inst. British Telecom. Eng., Vol. 2, Pt.3, p.92-95, July-Sept. 2001.

37. K.J. Vinoy, K.A. Jose, V.K. Varadan and V.V Varadan, "Hilbert curve fractal antenna: a small resonant antenna for VHF/UHF applications," Microwave and Optical Technology Letters, Vol. 29, No.4, p.215-19, 20 May 2001. 